\title{
Influence of asymmetry on bias behavior of spin torque
}

\author{
Y.-H. Tang, ${ }^{1}$ Nicholas Kioussis, ${ }^{1}$ Alan Kalitsov, ${ }^{1}$ W. H. Butler, ${ }^{2}$ and Roberto Car $^{3}$ \\ ${ }^{1}$ Department of Physics, California State University, Northridge, California 91330-8268, USA \\ ${ }^{2}$ MINT Center, University of Alabama, Tuscaloosa, Alabama 35487, USA \\ ${ }^{3}$ Department of Chemistry, Princeton University, Princeton, New Jersey 08544-0001, USA
}

(Received 9 November 2009; revised manuscript received 19 January 2010; published 23 February 2010)

\begin{abstract}
We report calculations, based on the tight-binding model and the nonequilibrium Keldysh formalism, of the effect of band-filling (BF) asymmetry between the ferromagnetic leads on the bias behavior of the spin torque and the tunneling magnetoresistance (TMR) in magnetic tunnel junctions. The underlying mechanism for the asymmetry-induced change in the bias dependence of TMR and the spin-transfer component, $T_{\|}$, is the interplay of charge and spin currents in the ferromagnetic (FM) and antiferromagnetic (AF) configurations. The BF asymmetry has a dramatic effect on the low-bias behavior of the fieldlike component, $T_{\perp}$, which can vary from linear to quadratic bias dependence with positive or negative curvature, thus reconciling the apparently contradictory experimental results. A general expression is derived relating $T_{\perp}$ with four independent nonequilibrium interlayer exchange couplings (NEIECs), $J_{\mathrm{FM}}^{\sigma \sigma^{\prime}}$, associated with the majority- and minority-spin channels, $\sigma, \sigma^{\prime}=\uparrow, \downarrow$, of the two leads solely in the FM configuration. The bias behavior of the NEIEC components can be selectively tuned with the BF of the free and pinned FM layers, thus opening a new avenue for controlling experimentally $T_{\perp}$.
\end{abstract}

DOI: 10.1103/PhysRevB.81.054437

PACS number(s): 85.75.-d, 72.25.-b, 73.40.Gk

\section{INTRODUCTION}

In recent years, the switching of magnetic states in noncollinear magnetic tunnel junctions (MTJ) by spin-polarized current via the spin torque has been the subject of intensive experimental $^{1-5}$ and theoretical ${ }^{6-10}$ research. This currentinduced magnetization reversal (CIMR) has promising applications in nonvolatile magnetic random access memories (MRAM) and magnetic sensors.

The spin torque can be decomposed into a fieldlike, $T_{\perp}$, and a spin-transfer, $T_{\|}$, components both orthogonal to the magnetization of the free ferromagnetic (FM) lead, where the first (latter) are perpendicular (parallel) to the plane of the magnetizations of the left (L) and right (R) FM leads, but with different bias behavior. For symmetric MTJ, we have predicted $^{8}$ that $T_{\|}$exerted on the right FM lead can be understood by the difference between the spin current in the antiferromagnetic (AF) and FM configurations. The bias dependence has an unusual nonmonotonic behavior and it may exhibit a sign reversal without a corresponding sign reversal of the bias or even a quadratic bias dependence. ${ }^{7,8}$

The bias dependence of $T_{\perp}$, directly related to the nonequilibrium interlayer exchange coupling (NEIEC), was predicted to be purely quadratic for symmetric MTJ with any band filling $(\mathrm{BF})$ and exchange splitting, with negative bias curvature. On the experimental side, Sankey et $a l .{ }^{3}$ and Kubota et $a{ }^{4}{ }^{4}$ have measured recently the bias dependence of $T_{\perp}$ in $\mathrm{Co}_{60} \mathrm{Fe}_{20} \mathrm{~B}_{20} / \mathrm{MgO} / \mathrm{Co}_{60} \mathrm{Fe}_{20} \mathrm{~B}_{20} \mathrm{MTJ}$, where the thickness of the free FM layer and the barrier are slightly different. They both find a quadratic bias dependence of $T_{\perp}$ in agreement with theoretical calculations, ${ }^{8-10}$ but with an apparently different bias curvature, presumably due to the different sign convention employed for the direction of both components of the spin torque. On the other hand, Petit et al. ${ }^{5}$ observed a linear bias dependence of $T_{\perp}$ in a $\mathrm{CoFe} / \mathrm{Al}_{2} \mathrm{O}_{3} / \mathrm{CoFe}$ MTJ. Thus, despite its importance in
CIMR applications, the bias behavior of $T_{\perp}$ remains unresolved.

It is known that the transport and the tunnel magnetoresistance (TMR) in MTJs can be asymmetric with regard to the polarity of the bias voltage. In symmetric MTJ the TMR has a maximum at zero bias and decreases with bias, ${ }^{11,12}$ and it can be selectively tuned by the BF and the exchange splitting in both left and right FM leads. ${ }^{13}$ The asymmetry in MTJ can arise from dissimilar electrodes, such as CoFeB/ $\mathrm{MgO} / \mathrm{NiFe}$ junctions, ${ }^{14}$ or from a physically asymmetric barrier under different oxidation conditions ${ }^{15,16}$ or when $\mathrm{Cu}$ diffuses into the $\mathrm{Al}_{2} \mathrm{O}_{3}$ interface in $\mathrm{Co} / \mathrm{Cu} / \mathrm{Co} / \mathrm{Al}_{2} \mathrm{O}_{3} / \mathrm{Co}$ MTJ during annealing. ${ }^{17}$ Interestingly, the effect of asymmetry has been observed ${ }^{18}$ even for nominally similar electrodes in $\mathrm{Co} / \mathrm{Al}_{2} \mathrm{O}_{3} / \mathrm{Co} \mathrm{MTJ}$, where one of the electrodes is textured primarily face-centered cubic (fcc) and the other is of mixed hexagonal-close-packed and fcc phases. This observation of asymmetry in nominally similar electrodes and the link to the crystalline phase is intriguing and implies that differences in electronic structure between dissimilar leads can cause a bias asymmetry in the TMR. The asymmetry shifts the maximum of the TMR toward finite bias and it may also inverse the sign of the TMR at higher bias. ${ }^{15,19-21}$ This can, in turn, be exploited in applications that require the junction to operate at maximum TMR under finite bias.

The effect of asymmetry on the bias behavior of both components of the spin torque in MTJ remains an unexplored area thus far both experimentally and theoretically, except for the recent calculations employing the free-electron gas model. ${ }^{10,22}$ The objective of this work is to present a comprehensive study of the effect of asymmetry between dissimilar FM leads on the bias behavior of TMR and spin torques, using the one-band tight-binding (TB) method with the nonequilibrium Keldysh formalism. We provide details of the analytical derivations and an extended discussion of the theory and predictions, presented briefly in Ref. 23. We 


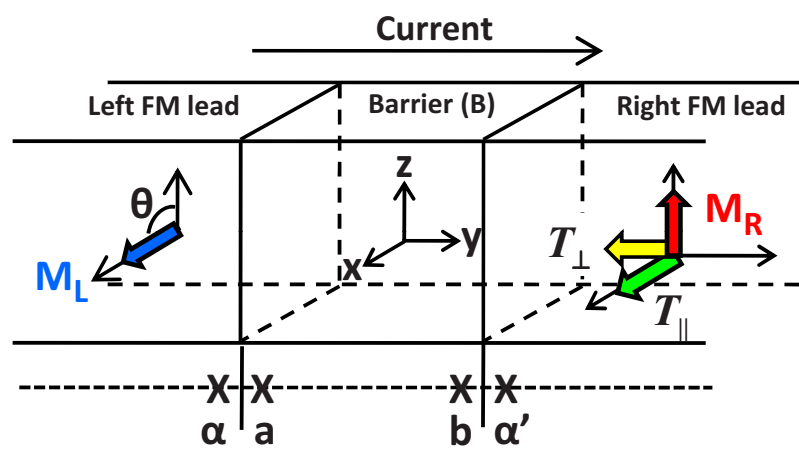

FIG. 1. (Color online) Schematic of MTJ, consisting of the left and right semi-infinite FM leads sandwiching the nonmagnetic barrier (B). The magnetization, $\mathbf{M}_{\mathbf{R}}$, of the right FM lead is along the $z$ axis, while that of the left FM lead, $\mathbf{M}_{\mathbf{L}}$, is rotated by the angle $\theta$ around the $y$ axis with respect to $\mathbf{M}_{\mathbf{R}} \cdot \alpha\left(\alpha^{\prime}\right)$ is the last (first) site of left (right) FM lead and $a(b)$ is the first (last) site in the barrier. The net spin-transfer, $T_{\|}$, and fieldlike, $T_{\perp}$, components of the spin torque on the right FM lead are along the $\hat{\mathbf{M}}_{\mathbf{R}} \times\left(\hat{\mathbf{M}}_{\mathbf{L}} \times \hat{\mathbf{M}}_{\mathbf{R}}\right)$ and $\left(\hat{\mathbf{M}}_{\mathbf{L}} \times \hat{\mathbf{M}}_{\mathbf{R}}\right)$ directions, respectively, where $\hat{\mathbf{M}}_{\mathbf{L}(\mathbf{R})}$ is the unit vector of the magnetization of the left (right) FM lead.

find that the bias behavior of $T_{\|}, T_{\perp}$, and TMR can be tuned selectively via the asymmetry in BF between the FM leads. Both $T_{\|}(V)$ and TMR $(V)$, including the sign reversal, can be explained by the bias behavior of the spin-polarized currents for the FM and AF configurations employing Brinkman's tunnel model ${ }^{24}$ for asymmetric barriers, generalized so as to take into account both spin channels. The BF asymmetry changes dramatically the low-bias behavior of $T_{\perp}$, which ranges from linear to quadratic bias dependence, with positive or negative bias curvature, $d^{2} T_{\perp}(V) / d V^{2}$. These results reconcile the origin of the experimental controversies, without invoking the recently proposed inelastic effects. ${ }^{25} \mathrm{We}$ present in detail the derivation of a general expression relating the bias behavior of $T_{\perp}$ in noncollinear MTJ with that of collinear (FM and AF) configurations. We demonstrate that the wide range of bias behavior of $T_{\perp}$ can be understood by the interplay of four independent NEIEC, $J_{\mathrm{FM}}^{\sigma \sigma^{\prime}}$, associated with the majority- and minority-spin channels, $\sigma, \sigma^{\prime}=\uparrow, \downarrow$, of the two leads solely in the FM configuration.

This paper is organized as follows. Section II describes the method used to model the asymmetric MTJ and to calculate the spin torques. Numerical results for the bias dependence of TMR, $T_{\|}$, and $T_{\perp}$, are presented in Secs. III A-III C, respectively. Finally, conclusions are summarized in Sec. IV.

\section{METHODOLOGY}

The MTJ, shown schematically in Fig. 1, consists of a left and right semi-infinite noncollinear FM leads, sandwiching an insulating barrier (B) containing $N=5$ atomic layers. The magnetization, $\mathbf{M}_{\mathbf{R}}$, of the right FM lead is along $z$ while that of the left FM lead, $\mathbf{M}_{\mathbf{L}}$, is rotated by the angle $\theta$ around the $y$ axis (normal to the $\mathrm{FM} / \mathrm{B}$ interface) with respect to $\mathbf{M}_{\mathbf{R}}$.

The Hamiltonian is described by the single-orbital simplecubic TB model ${ }^{13}$ with the spin-dependent on-site energies of the left FM lead, $\varepsilon_{\mathrm{L}}^{\uparrow}=1.2 \mathrm{eV}$ and $\varepsilon_{\mathrm{L}}^{\downarrow}=2.0 \mathrm{eV}$, the spin- independent on-site energy in the barrier, $\varepsilon_{\mathrm{B}}=5.4 \mathrm{eV}$, and the nearest-neighbor spin-independent hopping matrix element, $t=-0.4 \mathrm{eV}$, in all regions. This choice of TB parameters provided a realistic choice for systems based on magnetic transition metals and their alloys ${ }^{13}$ and gave an excellent agreement for the bias dependence of both $T_{\|}$and $T_{\perp}$ between our previous theoretical predictions ${ }^{8}$ and experiment. ${ }^{4}$ All calculations reported in this work were carried out at room temperature.

In asymmetric MTJ, both the majority and minority onsite energies of the right FM lead are rigidly shifted by $\delta$ $=\varepsilon_{\mathrm{R}}^{\uparrow(\downarrow)}-\varepsilon_{\mathrm{L}}^{\uparrow(\downarrow)}$, relative to those of the left FM lead, while, in order to minimize the TB parameters, the exchange splitting, $\Delta=\varepsilon_{\mathrm{L}(\mathrm{R})}^{\downarrow}-\varepsilon_{\mathrm{L}(\mathrm{R})}^{\uparrow}$, is fixed for both left and right FM leads. Experimentally, this could be achieved through alloying $\mathrm{Fe}(\mathrm{Co})$ with impurities which can shift the $\Delta_{1}$ band. Under external bias, $V$, the chemical potential of the right FM lead is shifted with respect to that of the left FM lead by, $\mu_{\mathrm{R}}$ $-\mu_{\mathrm{L}}=e V$, where $\mu_{\mathrm{L}}$ is fixed at the Fermi energy, $E_{F}=0 \mathrm{eV}$. For positive bias, the charge current flows from left to right along the $y$ axis in Fig. 1 .

The nonequilibrium Keldysh formalism ${ }^{26}$ is employed to calculate the net spin-transfer, $T_{\|}$, and fieldlike, $T_{\perp}$, components of the spin torque on the right FM lead, which can be simply expressed as the $x$ and $y$ components of the spin current density at the right $\mathrm{B} / \mathrm{FM}$ interface, ${ }^{8,13}$ respectively,

$$
T_{\|}=\frac{t}{16 \pi^{3}} \int \operatorname{Tr}\left[\left(\hat{G}_{\alpha^{\prime} b}^{<}-\hat{G}_{b \alpha^{\prime}}^{<}\right) \sigma_{x}\right] d E d \mathbf{k}_{\|}
$$

and

$$
T_{\perp}=\frac{t}{16 \pi^{3}} \int \operatorname{Tr}\left[\left(\hat{G}_{\alpha^{\prime} b}^{<}-\hat{G}_{b \alpha^{\prime}}^{<}\right) \sigma_{y}\right] d E d \mathbf{k}_{\|} .
$$

The spin-current density, $I_{z}^{(s)}$, along the $z$ axis and the charge current density, $I$, at the right $\mathrm{B} / \mathrm{FM}$ interface, are

$$
I_{z}^{(s)}=\frac{\hbar}{2 e}\left[I^{\uparrow}-I^{\downarrow}\right]=\frac{-t}{16 \pi^{3}} \int \operatorname{Tr}\left[\left(\hat{G}_{\alpha^{\prime} b}^{<}-\hat{G}_{b \alpha^{\prime}}^{<}\right) \sigma_{z}\right] d E d \mathbf{k}_{\|}
$$

and

$$
I=I^{\uparrow}+I^{\downarrow}=\frac{-e t}{8 \pi^{3} \hbar} \int \operatorname{Tr}\left[\hat{G}_{\alpha^{\prime} b}^{<}-\hat{G}_{b \alpha^{\prime}}^{<}\right] d E d \mathbf{k}_{\|},
$$

respectively. Here, $\hat{G}^{<}$is the $2 \times 2$ Keldysh Green's function matrix in spin space, and $\alpha\left(\alpha^{\prime}\right)$ is the last (first) site of the left (right) FM lead and $a(b)$ is the first (last) site in the barrier as shown in Fig. 1. $\sigma=\left(\sigma_{x}, \sigma_{y}, \sigma_{z}\right)$ is the vector of the $2 \times 2$ Pauli matrix, $\mathbf{k}_{\|}$is the transverse component of the wave vector, and the energy integral is over occupied states. Note that $T_{\|}, T_{\perp}$, and $I_{z}^{(s)}$ are calculated in $\mathrm{peV} / \square$ and $I$ is calculated in fA/ $\square$, where $\square$ refers to the interfacial unit area.

Applying the kinetic equation for the nonequilibrium 2 $\times 2$ Keldysh Green's function matrix in Eq. (15) of Ref. 13, 
results in a system of linear Dyson's equations for $\hat{G}_{\alpha^{\prime} b}^{<}$and $\hat{G}_{b \alpha^{\prime}}^{<}$of the form

$$
\begin{gathered}
\hat{G}_{\alpha^{\prime} b}^{<}=\hat{g}_{\alpha^{\prime} \alpha^{\prime}} t \hat{G}_{b b}^{<}+\hat{g}_{\alpha^{\prime} \alpha^{\prime}}^{<} t \hat{\widetilde{G}}_{b b}, \\
\hat{G}_{b b}^{<}=\hat{g}_{b a} t \hat{G}_{\alpha b}^{<}+\hat{g}_{b b} t \hat{G}_{\alpha^{\prime} b}^{<}, \\
\hat{G}_{\alpha b}^{<}=\hat{g}_{\alpha \alpha} t \hat{G}_{a b}^{<}+\hat{g}_{\alpha \alpha}^{<} t \hat{\widetilde{G}}_{a b}, \\
\hat{G}_{a b}^{<}=\hat{g}_{a a} t \hat{G}_{\alpha b}^{<}+\hat{g}_{a b} t \hat{G}_{\alpha^{\prime} b}^{<}
\end{gathered}
$$

and

$$
\begin{gathered}
\hat{G}_{b \alpha^{\prime}}^{<}=\hat{g}_{b a} t \hat{G}_{\alpha \alpha^{\prime}}^{<}+\hat{g}_{b b} t \hat{G}_{\alpha^{\prime} \alpha^{\prime}}^{<}, \\
\hat{G}_{\alpha \alpha^{\prime}}^{<}=\hat{g}_{\alpha \alpha} t \hat{G}_{a \alpha^{\prime}}^{<}+\hat{g}_{\alpha \alpha}^{<} t \hat{\tilde{G}}_{a \alpha^{\prime}}, \\
\hat{G}_{\alpha^{\prime} \alpha^{\prime}}^{<}=\hat{g}_{\alpha^{\prime} \alpha^{\prime}}^{<}+\hat{g}_{\alpha^{\prime} \alpha^{\prime}} t \hat{G}_{b \alpha^{\prime}}^{<}+\hat{g}_{\alpha^{\prime} \alpha^{\prime}}^{<}, \hat{\widetilde{G}}_{b \alpha^{\prime}}, \\
\hat{G}_{a \alpha^{\prime}}^{<}=\hat{g}_{a a} t \hat{G}_{\alpha \alpha^{\prime}}^{<}+\hat{g}_{a b} t \hat{G}_{\alpha^{\prime} \alpha^{\prime}}^{<} .
\end{gathered}
$$

Here, $\hat{g}(\hat{\tilde{g}})$ is the retarded (advanced) $2 \times 2$ Green's functions of the uncoupled regions and $\hat{g}_{i j}=g_{i j} \hat{I}$ is the retarded Green's functions of the isolated barrier, where $g_{i j}$ is real and $\hat{I}$ is the $2 \times 2$ unit matrix. The advanced Green's functions, $\hat{\widetilde{G}}$, of the coupled system are given by

$$
\begin{gathered}
\hat{\widetilde{G}}_{b \alpha^{\prime}}=\hat{\widetilde{G}}_{b b} t \hat{\tilde{g}}_{\alpha^{\prime} \alpha^{\prime}}, \quad \hat{\widetilde{G}}_{a \alpha^{\prime}}=\hat{\widetilde{G}}_{a b} t \hat{\tilde{g}}_{\alpha^{\prime} \alpha^{\prime}}, \\
\hat{\tilde{G}}_{a b}=\hat{g}_{a b}[\hat{\tilde{D}}]^{-1}, \\
\hat{\widetilde{G}}_{b b}=\left[\hat{g}_{b b}\left(\hat{I}-t^{2} \hat{\tilde{g}}_{\alpha \alpha} \hat{g}_{a a}\right)+\hat{g}_{a b} \hat{g}_{b a} t^{2} \hat{\tilde{g}}_{\alpha \alpha}\right][\hat{\tilde{D}}]^{-1},
\end{gathered}
$$

where

$$
\hat{\tilde{D}}=\left(\hat{I}-t^{2} \hat{\tilde{g}}_{\alpha \alpha} \hat{g}_{a a}\right)\left(\hat{I}-t^{2} \hat{\tilde{g}}_{\alpha^{\prime} \alpha^{\prime}} \hat{g}_{b b}\right)-t^{4} \hat{g}_{a b} \hat{g}_{b a} \hat{\tilde{g}}_{\alpha \alpha} \hat{\tilde{g}}_{\alpha^{\prime} \alpha^{\prime}}
$$

Solving the system of Eqs. (5)-(7) gives

$$
\begin{aligned}
\hat{G}_{\alpha^{\prime} b}^{<}-\hat{G}_{b \alpha^{\prime}}^{<}= & t^{3}[\hat{D} \hat{\tilde{D}}]^{-1} \hat{g}_{b a} \hat{g}_{a b} \times\left[\hat{g}_{\alpha^{\prime} \alpha^{\prime}} \hat{g}_{\alpha \alpha}^{<}+\hat{g}_{\alpha^{\prime} \alpha^{\prime}}^{<} \hat{\tilde{g}}_{\alpha \alpha}\right. \\
& \left.-\hat{g}_{\alpha \alpha}^{<} \hat{\tilde{g}}_{\alpha^{\prime} \alpha^{\prime}}-\hat{g}_{\alpha \alpha \alpha^{\prime}} \hat{g}_{\alpha^{\prime} \alpha^{\prime}}^{<}\right],
\end{aligned}
$$

where

$$
\begin{aligned}
\hat{g}_{\alpha \alpha\left(\alpha^{\prime} \alpha^{\prime}\right)}^{<} & =f_{\mathrm{L}(\mathrm{R})}\left[\hat{\tilde{g}}_{\alpha \alpha\left(\alpha^{\prime} \alpha^{\prime}\right)}-\hat{g}_{\alpha \alpha\left(\alpha^{\prime} \alpha^{\prime}\right)}\right] \\
& =-2 i f_{\mathrm{L}(\mathrm{R})} \operatorname{Im}\left\{\hat{g}_{\alpha \alpha\left(\alpha^{\prime} \alpha^{\prime}\right)}\right\} .
\end{aligned}
$$

$f_{\mathrm{L}}(E)$ and $f_{\mathrm{R}}(E-e V)$ are the Fermi-Dirac distribution functions of the left and right FM leads, respectively. In the limit of thick barrier, $\hat{D} \cdot \hat{\widetilde{D}} \simeq \hat{I}$, and the diagonal and off-diagonal spin-matrix elements of $\left(\hat{G}_{\alpha^{\prime} b}^{<}-\hat{G}_{b \alpha^{\prime}}^{<}\right)$in Eq. (9) can be recast in the form

$$
\begin{aligned}
G_{\alpha^{\prime} b}^{<\sigma \sigma}-G_{b \alpha^{\prime}}^{<\sigma \sigma} \approx & 2 i t^{3}\left|g_{a b}\right|^{2}\left[f_{\mathrm{L}} \operatorname{Im}\left\{g_{\alpha \alpha}^{\sigma \sigma}\right\}\left(\widetilde{g}_{\alpha^{\prime} \alpha^{\prime}}^{\sigma \sigma}-g_{\alpha^{\prime} \alpha^{\prime}}^{\sigma \sigma}\right)\right. \\
& \left.+f_{\mathrm{R}} \operatorname{Im}\left\{g_{\alpha^{\prime} \alpha^{\prime}}^{\sigma \sigma}\right\}\left(g_{\alpha \alpha}^{\sigma \sigma}-\widetilde{g}_{\alpha \alpha}^{\sigma \sigma}\right)\right]
\end{aligned}
$$

and

$$
\begin{aligned}
G_{\alpha^{\prime} b}^{<\sigma \bar{\sigma}}-G_{b \alpha^{\prime}}^{<\sigma \bar{\sigma}} \approx & 2 i t^{3}\left|g_{a b}\right|^{2}\left[f_{\mathrm{L}} \operatorname{Im}\left\{g_{\alpha \alpha}^{\sigma \bar{\sigma}}\right\}\left(\widetilde{g}_{\alpha^{\prime} \alpha^{\prime}}^{\bar{\sigma} \bar{\sigma}}-g_{\alpha^{\prime} \alpha^{\prime}}^{\sigma \sigma}\right)\right. \\
& \left.+f_{\mathrm{R}}\left(\operatorname{Im}\left\{g_{\alpha^{\prime} \alpha^{\prime}}^{\bar{\sigma} \bar{\sigma}}\right\} g_{\alpha \alpha}^{\sigma \bar{\sigma}}-\operatorname{Im}\left\{g_{\alpha^{\prime} \alpha^{\prime}}^{\sigma \sigma}\right\} \widetilde{g}_{\alpha \alpha}^{\sigma \bar{\sigma}}\right)\right],
\end{aligned}
$$

where $(\sigma, \bar{\sigma})=(\uparrow, \downarrow)$ are quantized along the $z$ axis. The retarded surface Green's functions for the isolated right and left FM leads with magnetizations $\mathbf{M}_{\mathbf{R}}$ and $\mathbf{M}_{\mathbf{L}}$ are

$$
\hat{g}_{\alpha^{\prime} \alpha^{\prime}}=\left[\begin{array}{cc}
g_{\alpha^{\prime} \alpha^{\prime}}^{\uparrow \uparrow} & 0 \\
0 & g_{\alpha^{\prime} \alpha^{\prime}}^{\downarrow \downarrow}
\end{array}\right] ; \quad \hat{g}_{\alpha \alpha}=\left[\begin{array}{cc}
g_{\alpha \alpha}^{\curlyvee} & 0 \\
0 & g_{\alpha \alpha}^{\searrow \searrow}
\end{array}\right] \text {, }
$$

respectively. Under rotation of $\mathbf{M}_{\mathbf{L}}$ by an angle $\theta$ along the direction of $\mathbf{M}_{\mathbf{R}}$, the surface Green's functions of the left FM lead can be written as

$$
\begin{gathered}
g_{\alpha \alpha}^{\uparrow \uparrow}=g_{\alpha \alpha}^{\nwarrow \nwarrow} \cos ^{2}(\theta / 2)+g_{\alpha \alpha}^{\searrow \searrow} \sin ^{2}(\theta / 2), \\
g_{\alpha \alpha}^{\downarrow \downarrow}=g_{\alpha \alpha}^{\nwarrow \nwarrow} \sin ^{2}(\theta / 2)+g_{\alpha \alpha}^{\searrow \searrow} \cos ^{2}(\theta / 2), \\
g_{\alpha \alpha}^{\uparrow \downarrow}=g_{\alpha \alpha}^{\downarrow \uparrow}=\frac{\sin (\theta)}{2}\left[g_{\alpha \alpha}^{\searrow \nwarrow}-g_{\alpha \alpha}^{\searrow \searrow}\right] .
\end{gathered}
$$

Here, $g_{\alpha \alpha}^{\uparrow \uparrow}\left(g_{\alpha \alpha}^{\downarrow \downarrow}\right)$ and $g_{\alpha \alpha}^{\nwarrow \Uparrow}\left(g_{\alpha \alpha}^{\searrow \downarrow}\right)$ are the surface Green's functions for the isolated left FM lead with $\mathbf{M}_{\mathbf{L}}$ quantized along the original and rotated $z$ axis. Note, that in the FM configuration, $g_{\alpha \alpha}^{\uparrow \uparrow}=g_{\alpha \alpha}^{\searrow \Uparrow}$ and $g_{\alpha \alpha}^{\downarrow \downarrow}=g_{\alpha \alpha}^{\searrow}$.

Substituting Eqs. (13) and (14) into Eqs. (11) and (12), $T_{\|}$, $T_{\perp}$, and $I_{z}^{(s)}$ in Eqs. (1)-(3) can be written in the form

$$
\begin{aligned}
T_{\|} \approx & \frac{t^{4} \sin \theta}{8 \pi^{3}} \int\left|g_{a b}\right|^{2} \times\left[f_{\mathrm{L}}(E)-f_{\mathrm{R}}(E-e V)\right] \\
& \times \operatorname{Im}\left\{g_{\alpha \alpha}^{\nwarrow \nwarrow}-g_{\alpha \alpha}^{\searrow \searrow}\right\} \times \operatorname{Im}\left\{g_{\alpha^{\prime} \alpha^{\prime}}^{\uparrow \uparrow}+g_{\alpha^{\prime} \alpha^{\prime}}^{\downarrow \downarrow}\right\} d \mathbf{k}_{\|} d E,
\end{aligned}
$$

$$
\begin{aligned}
T_{\perp} \approx & \frac{t^{4} \sin \theta}{8 \pi^{3}} \int d \mathbf{k}_{\|} d E\left|g_{a b}\right|^{2} \times\left[f_{\mathrm{L}}(E) \operatorname{Im}\left\{g_{\alpha \alpha}^{\nwarrow \nwarrow}-g_{\alpha \alpha}^{\searrow}\right\}\right. \\
& \times \operatorname{Re}\left\{g_{\alpha^{\prime} \alpha^{\prime}}^{\uparrow \uparrow}-g_{\alpha^{\prime} \alpha^{\prime}}^{\downarrow \downarrow}\right\}+f_{\mathrm{R}}(E-e V) \operatorname{Re}\left\{g_{\alpha \alpha}^{\searrow \uparrow}-g_{\alpha \alpha}^{\searrow}\right\} \\
& \left.\times \operatorname{Im}\left\{g_{\alpha^{\prime} \alpha^{\prime}}^{\uparrow \uparrow}-g_{\alpha^{\prime} \alpha^{\prime}}^{\downarrow \downarrow}\right\}\right],
\end{aligned}
$$

and 


$$
\begin{aligned}
I_{z}^{(s)} \approx & \frac{-t^{4} \sin \theta}{8 \pi^{3}} \int d \mathbf{k}_{\|} d E\left|g_{a b}\right|^{2}\left[f_{\mathrm{L}}(E)-f_{\mathrm{R}}(E-e V)\right] \\
& \times\left[\operatorname { I m } \{ g _ { \alpha ^ { \prime } \alpha ^ { \prime } } ^ { \uparrow \uparrow } \} \left[\operatorname{Im}\left\{g_{\alpha \alpha}^{\nwarrow}\right\} \cos ^{2}\left(\frac{\theta}{2}\right)\right.\right. \\
& \left.+\operatorname{Im}\left\{g_{\alpha \alpha}^{\searrow}\right\} \sin ^{2}\left(\frac{\theta}{2}\right)\right]-\operatorname{Im}\left\{g_{\alpha^{\prime} \alpha^{\prime}}^{\downarrow \downarrow}\right\}\left[\operatorname{Im}\left\{g_{\alpha \alpha}^{\nwarrow \nwarrow}\right\} \sin ^{2}\left(\frac{\theta}{2}\right)\right. \\
& \left.\left.+\operatorname{Im}\left\{g_{\alpha \alpha}^{\searrow \searrow}\right\} \cos ^{2}\left(\frac{\theta}{2}\right)\right]\right] .
\end{aligned}
$$

Comparison between Eqs. (15) and (17), yields the general expression

$$
T_{\|}(\theta)=\frac{I_{z}^{(s)}(\mathrm{AF})-I_{z}^{(s)}(\mathrm{FM})}{2} \times \sin \theta,
$$

for the noncollinear spin-transfer torque, $T_{\|}$, in asymmetric MTJ, in terms of the spin currents, $I_{z}^{(s)}$, for the FM and AF collinear configurations, originally predicted by Theodonis et $a l{ }^{8}$ only for symmetric MTJ. This general expression demonstrates that the bias dependence of noncollinear $T_{\|}$can be decomposed as the interplay between four independent nonequilibrium spin-polarized current densities, $I_{z}^{\sigma}$, solely in the FM and AF collinear configurations.

Since $T_{\perp}$ is directly related to the NEIEC, ${ }^{7,27}$ Eq. (16) yields another general expression for the fieldlike component of the spin torque, namely,

$$
T_{\perp}(\theta)=-\left[J_{\mathrm{FM}}^{\uparrow \uparrow}+J_{\mathrm{FM}}^{\downarrow \downarrow}-J_{\mathrm{FM}}^{\downarrow \uparrow}-J_{\mathrm{FM}}^{\uparrow \downarrow}\right] \times \sin \theta,
$$

where the NEIEC, $J_{\mathrm{FM}}^{\sigma \sigma^{\prime}}$, between the $\sigma$ - and $\sigma^{\prime}$-spin states in the left and right leads in the FM configuration are given by

$$
\begin{aligned}
-J_{\mathrm{FM}}^{\sigma \sigma^{\prime}} \approx & \frac{t^{4}}{8 \pi^{3}} \int g_{b a} g_{a b}\left[f_{\mathrm{L}}(E) \operatorname{Im}\left\{g_{\alpha \alpha}^{\sigma \sigma}\right\} \operatorname{Re}\left\{g_{\alpha^{\prime} \alpha^{\prime}}^{\sigma^{\prime} \sigma^{\prime}}\right\}\right. \\
& \left.+f_{\mathrm{R}}(E-e V) \operatorname{Re}\left\{g_{\alpha \alpha}^{\sigma \sigma}\right\} \operatorname{Im}\left\{g_{\alpha^{\prime} \alpha^{\prime}}^{\sigma^{\prime} \sigma^{\prime}}\right\}\right] d \mathbf{k}_{\|} d E .
\end{aligned}
$$

Equation (19) demonstrates that the bias dependence of $T_{\perp}$ in the noncollinear configuration can be expressed in terms of four independent NEIECs solely in the FM $(\theta=0)$ collinear configuration. Our numerical results demonstrate that both Eqs. (18) and (19) are independent of the details of electronic structures. The $\sin \theta$ dependence of both $T_{\|}$and $T_{\perp}$ agrees with previous experimental ${ }^{3,4}$ and theoretical findings.., 8

\section{RESULTS AND DISCUSSION}

\section{A. Bias behavior of TMR}

In Fig. 2(a), we present the bias behavior of TMR $=\frac{I(\mathrm{FM})-I(\mathrm{AF})}{I(\mathrm{AF})}$ for various values of the asymmetry energy parameter $\delta$. For symmetric MTJ $(\delta=0.0 \mathrm{eV})$, the TMR decreases with increasing bias exhibiting a maximum at zero bias $\left(V_{\max }=0 \mathrm{~V}\right)$ and changes sign at the critical bias, $V_{c}$ $\sim \pm 0.55 \mathrm{~V}$. For $\delta>0(<0)$, where the barrier height at the right $\mathrm{B} / \mathrm{FM}$ interface is smaller (larger) than that of the left $\mathrm{B} / \mathrm{FM}$ interface, the bias behavior of the TMR is shifted toward negative (positive) bias, giving rise to a negative (posi-

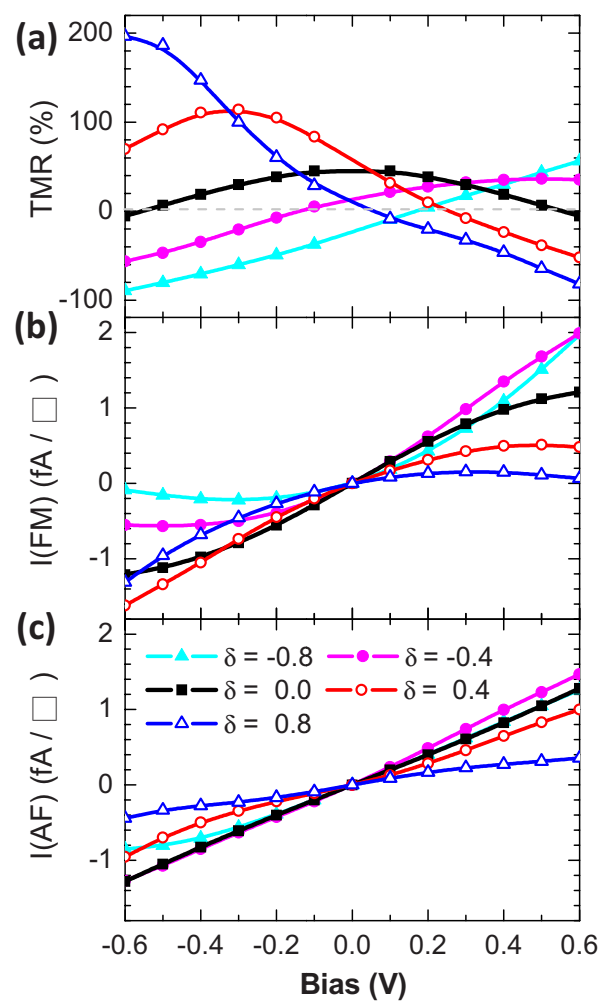

FIG. 2. (Color online) Bias dependence of (a) tunnel magnetoresistance, TMR, and of the charge current density in the (b) FM and (c) AF configurations, for various values of the asymmetry energy parameter $\delta$.

tive) $V_{\max }$ and a shift of $V_{c}$ toward negative (positive) bias. These findings are in agreement with experiment ${ }^{15}$ in asymmetric $\mathrm{Py} / \mathrm{AlO}_{x} / \mathrm{ZrO}_{y} / \mathrm{Py} \mathrm{MTJ}$, where $V_{\max }$ was found to decrease with increasing $\mathrm{Zr}$ thickness, due to the decrease in the barrier height of the $\mathrm{ZrO}_{y} / \mathrm{Py}$ interface. Furthermore, our results are in agreement with theoretical calculations ${ }^{19} \mathrm{em}-$ ploying the free-electron model, which found that $V_{c}$ decreases with decreasing barrier height. The offset of TMR for $\delta>0(<0)$ and the increase in TMR with the absolute value of bias in the negative (positive) low-bias region suggest that the asymmetry effect may be beneficial for practical applications in MRAM.

In order to elucidate the bias behavior of the TMR, we show in Figs. 2(b) and 2(c) the charge current densities, $I(\mathrm{FM})$ and $I(\mathrm{AF})$, in the $\mathrm{FM}$ and $\mathrm{AF}$ configurations, respectively, as a function of $V$. Applying Brinkman's tunnel model $^{24}$ for asymmetric barrier, the bias dependence of $I^{\sigma}$ can be expressed as

$$
I^{\sigma}=g_{1}\left(\bar{\phi}^{\sigma}\right) V-g_{2}\left(\bar{\phi}^{\sigma}\right) \Delta \phi^{\sigma} V^{2}+O\left(V^{3}\right),
$$

where $\bar{\phi}^{\sigma}=\left[\phi_{\mathrm{L}}^{\sigma}+\phi_{\mathrm{R}}^{\sigma}\right] / 2$ and $\Delta \phi^{\sigma}=\phi_{\mathrm{L}}^{\sigma}-\phi_{\mathrm{R}}^{\sigma}$ are the average barrier height and barrier asymmetry for the $\sigma$-spin state, respectively, $\phi_{\mathrm{L}(\mathrm{R})}^{\sigma}$ is the barrier height at the left (right) $\mathrm{B} / \mathrm{FM}$ interface from the bottom of the $\sigma$-spin band, and $g_{1}$ and $g_{2}$ are functions of the average barrier height and barrier asymmetry. For the FM configuration, shown in the top panel of Fig. $3, \bar{\phi}^{\uparrow}=\phi_{1}-\frac{\delta}{2}, \bar{\phi}^{\downarrow}=\phi_{2}-\frac{\delta}{2}$, and $\Delta \phi^{\uparrow(\downarrow)}=\delta$, yielding 


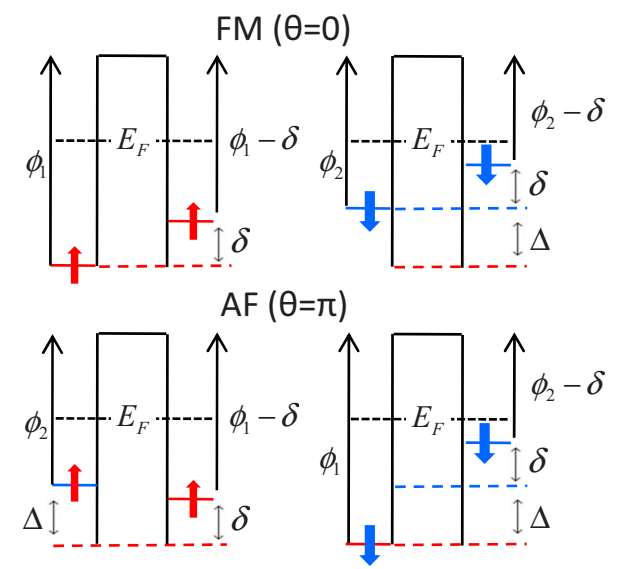

FIG. 3. (Color online) Schematic energy profiles of the majority and minority on-site energy levels in the FM (top) and AF (bottom) configurations. $\phi_{1}\left(\phi_{2}\right)$ denotes the barrier height from the bottom of the majority (minority) band of the left lead; $\delta=\varepsilon_{\mathrm{R}}^{\uparrow(\downarrow)}-\varepsilon_{\mathrm{L}}^{\uparrow(\downarrow)}$ and $\Delta=\varepsilon_{\mathrm{L}(\mathrm{R})}^{\downarrow}-\varepsilon_{\mathrm{L}(\mathrm{R})}^{\uparrow}$ are the asymmetry energy parameter and exchange splitting, respectively.

$$
\begin{aligned}
I(\mathrm{FM})= & I^{\uparrow}(\mathrm{FM})+I^{\downarrow}(\mathrm{FM})=\left[g_{1}\left(\phi_{1}-\delta / 2\right)+g_{1}\left(\phi_{2}-\delta / 2\right)\right] V \\
& -\delta\left[g_{2}\left(\phi_{1}-\delta / 2\right)+g_{2}\left(\phi_{2}-\delta / 2\right)\right] V^{2}+O\left(V^{3}\right),
\end{aligned}
$$

where $\phi_{1}\left(\phi_{2}\right)$ denotes the barrier height from the bottom of the majority (minority) band of the left lead. On the other hand, for the AF configuration, shown in the lower panel of Fig. $3, \bar{\phi}^{\uparrow(\downarrow)}=\left[\phi_{1}+\phi_{2}-\delta\right] / 2$ and $\Delta \phi^{\uparrow(\downarrow)}= \pm\left(\phi_{2}-\phi_{1}\right)+\delta$, and

$$
\begin{aligned}
I(\mathrm{AF})= & I^{\uparrow}(\mathrm{AF})+I^{\downarrow}(\mathrm{AF})=2 g_{1}\left(\left[\phi_{1}+\phi_{2}-\delta\right] / 2\right) V \\
& -2 \delta g_{2}\left(\left[\phi_{1}+\phi_{2}-\delta\right] / 2\right) V^{2}+O\left(V^{3}\right) .
\end{aligned}
$$

Since $g_{1}\left(\bar{\phi}^{\sigma}\right)$ and $g_{2}\left(\bar{\phi}^{\sigma}\right)$ are positive, both $I(\mathrm{FM})$ and $I(\mathrm{AF})$ have positive slopes and negative (positive) bias curvatures for $\delta>0(<0)$. Since for asymmetric MTJ the linear terms in Eqs. (22) and (23) for the $I(\mathrm{FM})$ and $I(\mathrm{AF})$, respectively, do not cancel out, the bias behavior of the TMR is not purely quadratic, leading to the offset of the TMR and the shifts of both $V_{c}$ and $V_{\max }$.

\section{B. Bias behavior of $T_{\|}$}

In Fig. 4(a) we present the bias dependence of the spintransfer torques, $T_{\|}\left(\theta=\frac{\pi}{2}\right)$, for various values of $\delta$. The solid points and curves correspond to $T_{\|}$calculated from Eqs. (1) and (15), respectively. The agreement between these two computational approaches demonstrates that the expression (18) is general and is also valid for asymmetric MTJ. For symmetric MTJ $(\delta=0.0 \mathrm{eV}), T_{\|}$has a nonmonotonic bias behavior with a minimum value at $V_{\min } \sim 0.17 \mathrm{~V}$ and changes sign at the bias reversal $V_{\text {rev }} \sim 0.34 \mathrm{~V}$. The asymmetry effect preserves the nonmonotonic bias dependence of $T_{\|}$, but shifts both $V_{\min }$ and $V_{\text {rev }}$ toward negative (positive) bias values for $\delta>0(\delta<0)$. This suggests that one can control the CIMS in MTJ by varying the asymmetry between two FM leads.

In order to understand the underlying mechanism of $T_{\|}(V)$ in Eq. (18), we present in Figs. 4(b) and 4(c) the bias depen-

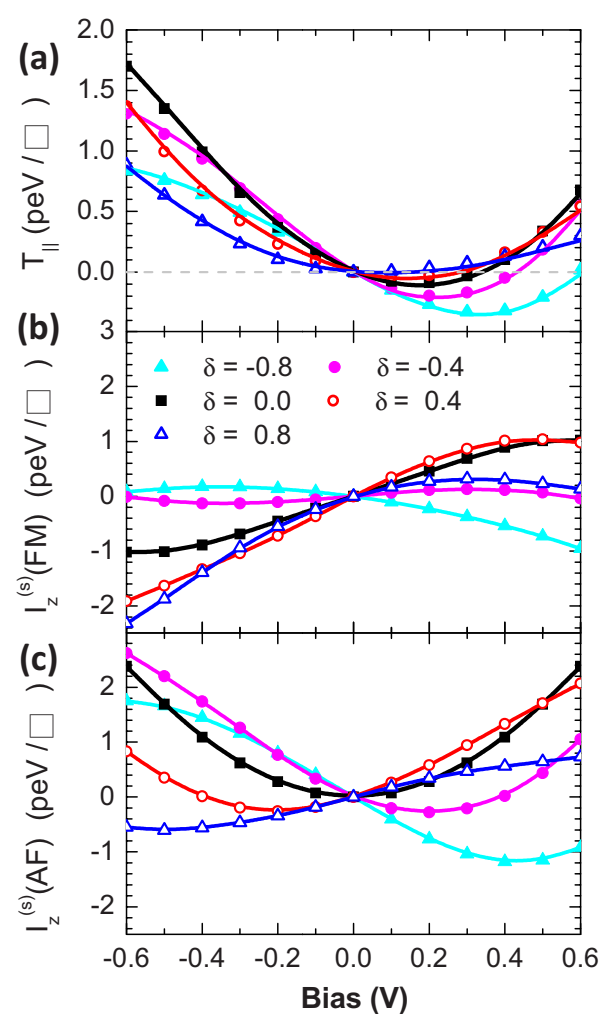

FIG. 4. (Color online) Bias dependence of (a) $T_{\|}(\theta=\pi / 2)$, and the spin-current density (b) $I_{z}^{(s)}(\mathrm{FM})$ in the FM configuration, and (c) $I_{z}^{(s)}(\mathrm{AF})$ in the $\mathrm{AF}$ configuration, for different values of the asymmetry energy parameter $\delta$. The solid points and curves in (a) correspond to $T_{\|}$calculated from Eqs. (1) and (15), respectively.

dence of the spin currents $I_{z}^{(s)}(\mathrm{FM})$ and $I_{z}^{(s)}(\mathrm{AF})$ for the FM and AF configurations, respectively. Using Eq. (21) the spin currents for the FM and AF configurations can be written in the form

$$
\begin{aligned}
I_{z}^{(s)}(\mathrm{FM})= & \frac{\hbar}{2 e}\left\{\left[g_{1}\left(\phi_{1}-\delta / 2\right)-g_{1}\left(\phi_{2}-\delta / 2\right)\right] V\right. \\
& \left.-\delta\left[g_{2}\left(\phi_{1}-\delta / 2\right)-g_{2}\left(\phi_{2}-\delta / 2\right)\right] V^{2}\right\}
\end{aligned}
$$

and

$$
I_{z}^{(s)}(\mathrm{AF})=\frac{\hbar}{e}\left\{\left(\phi_{1}-\phi_{2}\right) g_{2}\left[\left(\phi_{1}+\phi_{2}-\delta\right) / 2\right] V^{2}\right\} .
$$

The slope and bias curvature of $I_{z}^{(s)}(\mathrm{FM})$ depend both on the bias and barrier asymmetry $\delta$. On the other hand, $I_{z}^{(s)}(\mathrm{AF})$ exhibits a quadratic bias dependence with positive curvature. The shift in $V_{\min }$ of $I_{z}^{(s)}(\mathrm{AF})$ in Fig. 4(c) implies the presence of nonvanishing higher-order bias terms, giving rise to the negative (positive) offset of $I_{z}^{(s)}(\mathrm{AF})$ for $\delta>0(\delta<0)$. Therefore, the interplay between the bias dependence of $I_{z}^{(s)}(\mathrm{FM})$ and $I_{z}^{(s)}(\mathrm{AF})$ is responsible for the variation in the nonmonotonic bias dependence of $T_{\|}$and the offset in the bias behavior of $T_{\|}$with asymmetry.

\section{Bias behavior of $T_{\perp}$}

In Fig. 5 we present the bias behavior of $T_{\perp}(\theta=\pi / 2)$ for various values of $\delta$. The solid points and curves represent $T_{\perp}$ 


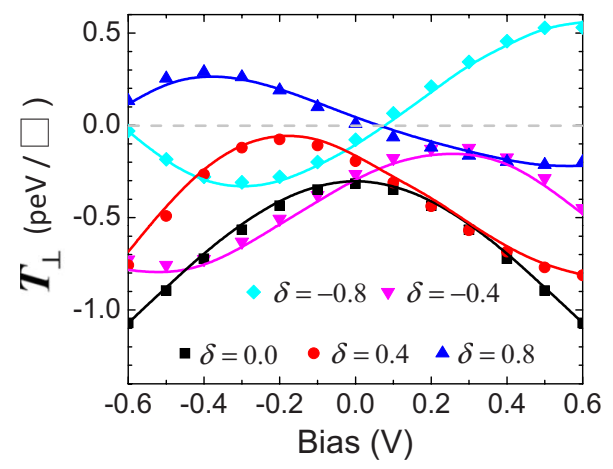

FIG. 5. (Color online) Bias dependence of $T_{\perp}(\theta=\pi / 2)$ for various values of the asymmetry energy parameter $\delta$. The solid points and curves correspond to $T_{\perp}$ calculated from Eqs. (2) and (19), respectively.

calculated from Eqs. (2) and (19), respectively. The good agreement demonstrates that the bias behavior of the noncollinear $T_{\perp}$ can be understood in terms of the interplay of four NEIECs, $J_{\mathrm{FM}}^{\sigma \sigma^{\prime}}$, associated with the majority- and minorityspin channels, $\sigma, \sigma^{\prime}=\uparrow, \downarrow$, of the two leads solely in the FM configuration. The $\mathrm{BF}$ asymmetry changes dramatically $T_{\perp}(V)$ : for the symmetric case $(\delta=0.0 \mathrm{eV})$ the bias behavior is purely quadratic with $d^{2} T_{\perp}(V) / d V^{2}<0$; for $\delta=0.4 \mathrm{eV}$ $(-0.4) \mathrm{eV}$ the maximum is shifted toward the negative (positive) bias. Note, that in both cases $T_{\perp}(V)$ does not reverse sign for any bias. On the other hand, for $\delta=0.8 \mathrm{eV}$ $(-0.8) \mathrm{eV} T_{\perp}$ exhibits a linear bias behavior at low bias $(|V| \leq 0.4 \mathrm{~V})$ and reverses sign with bias.

In the right panels of Figs. 6(a)-6(c) we show the bias dependence of the NEIECs, $J_{\mathrm{FM}}^{\sigma \sigma^{\prime}}$, associated with the spin channels, $\sigma, \sigma^{\prime}=\uparrow, \downarrow$, of the two leads in the FM configuration, for $\delta=0.0,+0.4$, and $-0.4 \mathrm{eV}$, respectively. For comparison we also show the bias behavior (black curves) of $T_{\perp}(V)$. The left (a)-(c) panels show schematic energy profiles of the bottom of the majority-(minority-) spin energy bands of the right and left leads, denoted by lower (higher) horizontal solid lines, for $\delta=0.0,+0.4$, and $-0.4 \mathrm{eV}$, respectively. The asymmetry energy parameter and the exchange spin splitting are denoted by $\delta$ and $\Delta$, respectively. The dashed lines represent the NEIECs, $J_{\mathrm{FM}}^{\sigma \sigma^{\prime}}$, and the solid horizontal line indicates the Fermi energy in equilibrium.

For the symmetric MTJ in Fig. 6(a), both $-J_{\mathrm{FM}}^{\uparrow \uparrow}$ and $-J_{\mathrm{FM}}^{\downarrow \downarrow}$ exhibit quadratic bias behavior with maximum value at zero bias, due to the same BF of the left and right leads. The different curvatures and zero-bias values are due to the different BFs of the $\uparrow$-spin and $\downarrow$-spin bands. On the other hand, $-J_{\mathrm{FM}}^{\uparrow \downarrow(\downarrow \uparrow)}$ exhibits an asymmetric bias behavior with their maximum shifted toward negative (positive) bias, due to the smaller (larger) BF of the minority (majority) bands of the right FM lead compared to the majority (minority) bands of the left FM lead. Interestingly, even though each NEIEC has a complex bias dependence, their algebraic sum has a purely quadratic behavior. For asymmetric MTJ $(\delta= \pm 0.4 \mathrm{eV})$, shown in Figs. 6(b) and 6(c), the smaller or larger BF of the right FM lead gives rise to an asymmetric bias dependence of the four NEIECs and hence of $T_{\perp}$. Thus, we predict that the bias, $V_{\max }^{\sigma \sigma^{\prime}}$, at which $J_{\mathrm{FM}}^{\sigma \sigma^{\prime}}$ reaches its first maximum value,

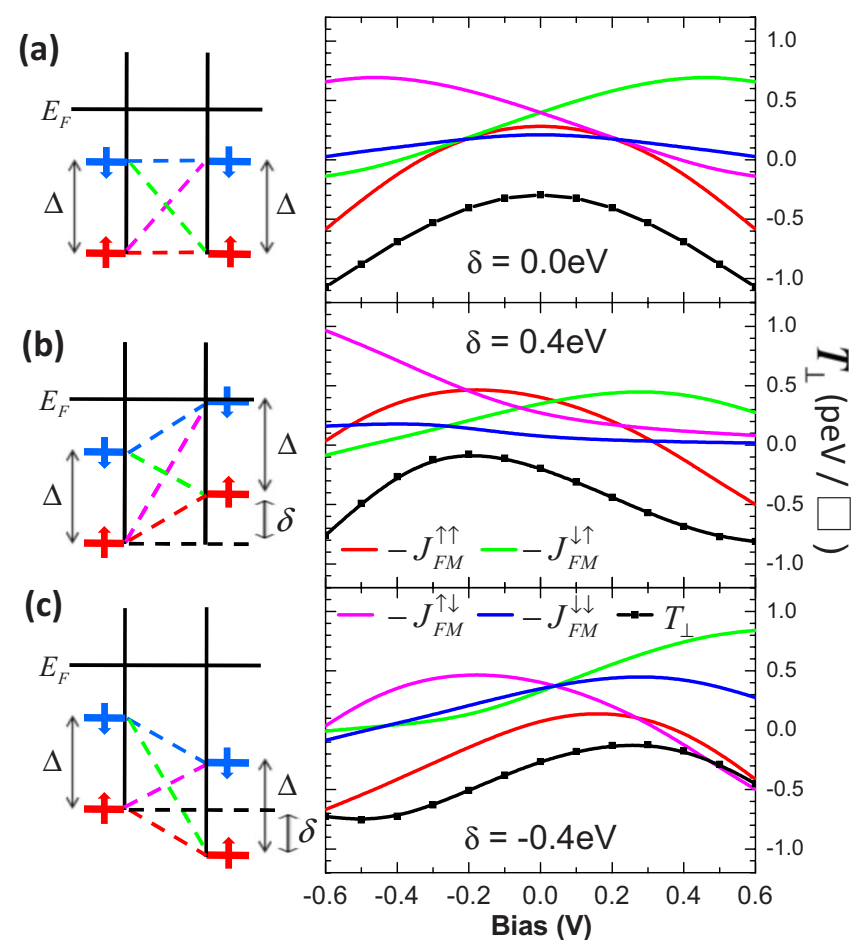

FIG. 6. (Color online) Left panels: schematic energy profile of the bottom of the majority-(minority-) energy bands on the right and left leads, denoted by lower (higher) horizontal solid lines, in FM configuration for various values of the asymmetry energy parameter $\delta$. The exchange splitting is denoted by $\Delta$, and the dashed lines represent the NEIECs. Right panels: bias dependence of $J_{\mathrm{FM}}^{\sigma \sigma^{\prime}}$ $\left(\sigma, \sigma^{\prime}=\uparrow, \downarrow\right)$ and $T_{\perp}(\theta=\pi / 2)$, for $\delta=0.0,0.4$, and $-0.4 \mathrm{eV}$, respectively.

can be controlled by varying the relative BF between the $\sigma$ and $\sigma^{\prime}$ bands of the left and right leads. Namely,

$$
V_{\max }^{\sigma \sigma^{\prime}} \begin{cases}\leq 0 & \text { if } \epsilon_{\mathrm{L}}^{\sigma} \leq \epsilon_{\mathrm{R}}^{\sigma^{\prime}} \text { or } n_{\mathrm{L}}^{\sigma} \geq n_{\mathrm{R}}^{\sigma^{\prime}}, \\ \geq 0 & \text { if } \epsilon_{\mathrm{L}}^{\sigma} \geq \epsilon_{\mathrm{R}}^{\sigma^{\prime}} \text { or } n_{\mathrm{L}}^{\sigma} \leq n_{\mathrm{R}}^{\sigma^{\prime}} .\end{cases}
$$

Here, $n_{\mathrm{L}(\mathrm{R})}^{\sigma}$ is the $\mathrm{BF}$ of the $\sigma$ band of the left (right) lead. Furthermore, $\left|V_{\max }^{\sigma \sigma^{\prime}}\right|$ increases as the difference $\left|\epsilon_{\mathrm{L}}^{\sigma}-\epsilon_{\mathrm{R}}^{\sigma^{\prime}}\right|$ or $\left|n_{\mathrm{L}}^{\sigma}-n_{\mathrm{R}}^{\sigma^{\prime}}\right|$ increases. These predictions, valid for any value of $\Delta$ and $\delta$, may serve as simple guiding rules for future experimental applications in the writing process in MRAM via controlled fabrication of the FM leads. ${ }^{23,28}$

In Figs. 7(a)-7(c) we show the energy dependence of the left $(\mathrm{L})$ and right $(\mathrm{R})$ reservoir contributions, $T_{\perp}^{\mathrm{L}}(E)$ and $T_{\perp}^{\mathrm{R}}(E)$, respectively, to the net $T_{\perp}=T^{\mathrm{L}}+T_{\perp}^{\mathrm{R}}$, for different values of $V$ and $\delta$. Here, $T_{\perp}^{\mathrm{L}}(E)$ and $T_{\perp}^{\mathrm{R}}(E)$ correspond to the two terms of the integrand in Eq. (16) (integrated over $\mathbf{k}_{\|}$), proportional to $f_{\mathrm{L}}(E)$ and $f_{\mathrm{R}}(E-e V)$, respectively. The red (blue) shaded area denotes those energies within the bias window between $\mu_{\mathrm{L}}=0 \mathrm{eV}$ and $\mu_{\mathrm{R}}$ under $0.2 \mathrm{~V}(-0.2 \mathrm{~V})$ bias. Since $T_{\perp}$ is directly related to the net NEIEC, $J$ $=[E(\mathrm{FM})-E(\mathrm{AF})] / 2,{ }^{27}$ where $E(\mathrm{FM})$ and $E(\mathrm{AF})$ are the total energies of the FM and AF configurations, respectively, the entire energy spectrum of occupied states contributes to $T_{\perp}$. In contrast, only those energy states within the red or 


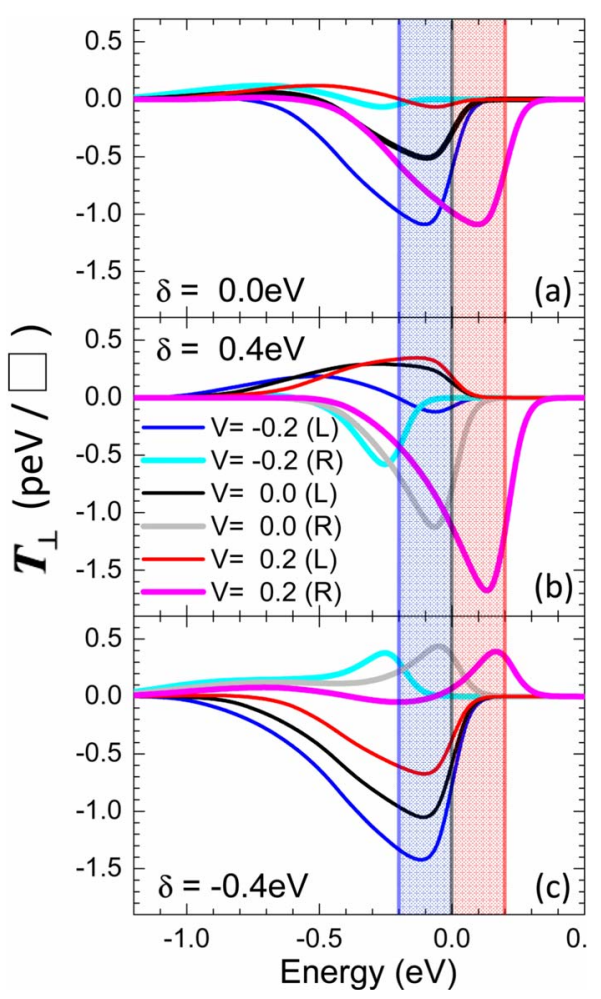

FIG. 7. (Color online) Energy dependence of the L and R lead contributions to $T_{\perp}$ with $V=-0.2,0.0$, and $0.2 \mathrm{~V}$ for (a) $\delta$ $=0.0 \mathrm{eV}$, (b) $\delta=0.4 \mathrm{eV}$, and (c) $\delta=-0.4 \mathrm{eV}$, respectively. The blue, black, and red vertical lines refer to $\mu_{\mathrm{R}}$, for the above bias values, while $\mu_{\mathrm{L}}$ is fixed at $E_{F}=0 \mathrm{eV}$.

blue shaded area are important for $T_{\|}$, since $T_{\|}$is directly related to the spin-current density in Eq. (18).

At zero bias, for symmetric MTJ $(\delta=0.0 \mathrm{eV})$ in Fig. 7(a), $T_{\perp}^{\mathrm{L}}(E)=T_{\perp}^{\mathrm{R}}(E)$ exhibit a broad negative dip at about $-0.1 \mathrm{eV}$. On the other hand, for $\delta>0(<0)$, shown in Fig. 7(b) [Fig. 7(c)], where one decreases (increases) the BF of the right $\mathrm{FM}$ lead, there is a dramatic change in $T_{\perp}^{\mathrm{L}}(E)$ $\left[T_{\perp}^{\mathrm{R}}(E)\right]$, which becomes positive over the entire occupied spectrum region, while the negative dip around $E_{F}$ of $T_{\perp}^{\mathrm{R}}(E)$ $\left[T_{\perp}^{\mathrm{L}}(E)\right]$ becomes more pronounced. This change in $T_{\perp}^{\mathrm{L}(\mathrm{R})}(E)$ can be understood in terms of the zero-bias $\mathbf{k}_{\|}$-resolved $T_{\perp}^{\mathrm{L}(\mathrm{R})}$, integrated over occupied states, which is shown in Figs. $8(\mathrm{a})-8(\mathrm{e})$ for various values of $\delta$. For symmetric MTJ, $T_{\perp}^{\mathrm{L}(\mathrm{R})}$ exhibits a negative ring around the $\Gamma$ point and becomes positive at $\mathbf{k}_{\|} \sim \pi / 2$. On the other hand, for $\delta>0(<0)$, both the positive contribution of $T_{\perp}^{\mathrm{L}}\left(T_{\perp}^{\mathrm{R}}\right)$ and the negative contribution of $T_{\perp}^{\mathrm{R}}\left(T_{\perp}^{\mathrm{L}}\right)$ around the $\Gamma$ point have smaller (larger) area due to the smaller (larger) BF of the right FM lead.

The application of positive (negative) bias for symmetric MTJ in Fig. 7(a) renders the $T_{\perp}^{\mathrm{L}}(E)\left[T_{\perp}^{\mathrm{R}}(E)\right]$ contributions positive over the low-energy region of the occupied spectrum $\left(E-E_{F} \leq-0.3 \mathrm{eV}\right)$, while it remains negative in the energy window of about $0.3 \mathrm{eV}$ below the Fermi energy. Furthermore, the application of positive (negative) bias for symmetric MTJ induces a large enhancement of the negative dip of $T_{\perp}^{\mathrm{R}}(E)\left[T_{\perp}^{\mathrm{L}}(E)\right]$ in the energy range both within and outside the bias window. The fact that $T_{\perp}^{\mathrm{R}}(E)$ decreases stronger than $T_{\perp}^{\mathrm{L}}(E)$ increases under positive bias, results in the decrease in
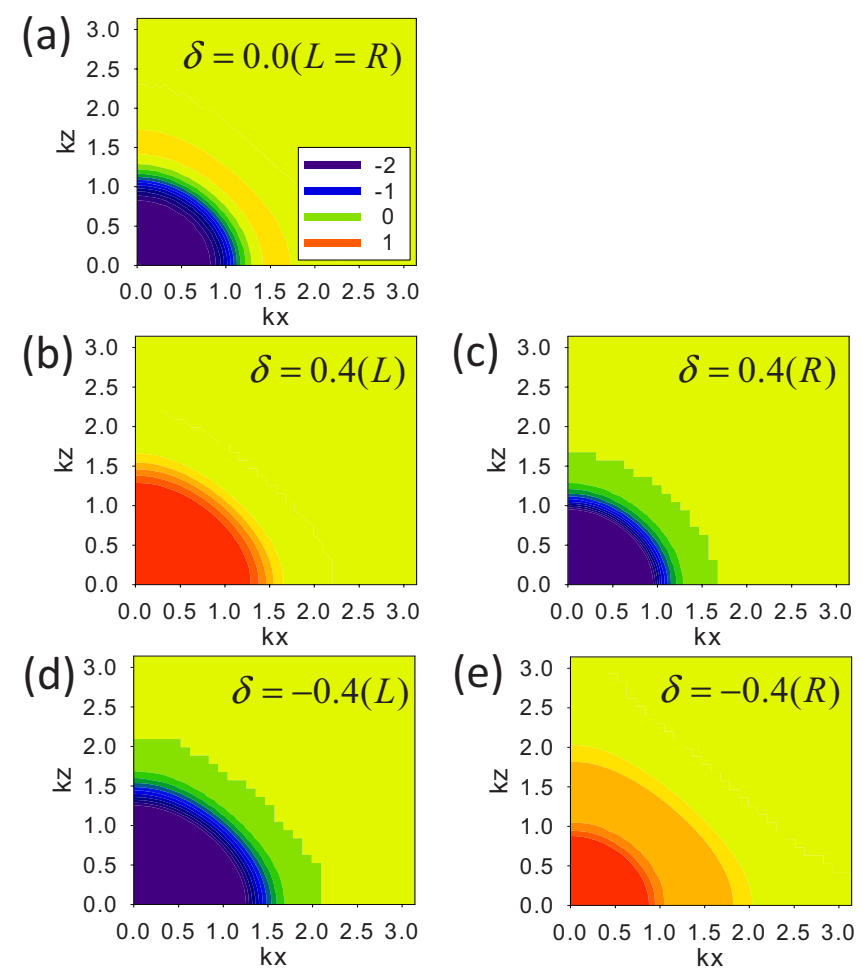

FIG. 8. (Color online) Zero-bias $\mathbf{k}_{\|}$-resolved L and R contributions to $T_{\perp}$ integrated over occupied states for (a) $\delta=0.0 \mathrm{eV}$ (L $=\mathrm{R}$ ), (b) and (c) $\delta=0.4 \mathrm{eV}$, and (d) and (e) $\delta=-0.4 \mathrm{eV}$, respectively.

$T_{\perp}$ with bias and hence $d^{2} T_{\perp}(V) / d V^{2}<0$ in Fig. 5. Thus, the effect of bias on $T_{\perp}^{\mathrm{L}}(E)$ and $T_{\perp}^{\mathrm{R}}(E)$ is analogous to that of the BF. For $\delta=0.4 \mathrm{eV}$ [Fig. 7(b)], the positive (negative) bias enhances (reduces) the negative $\operatorname{dip}$ of $T_{\perp}^{\mathrm{R}}(E)$ within the red (blue) shaded area shifting it to higher (lower) energy. The bias effect is weaker on the energy dependence of $T_{\perp}^{\mathrm{L}}(E)$. These two effects lead to the monotonic decrease in $T_{\perp}$ with bias in the low-bias regime in Fig. 5. In contrast, for $\delta=$ $-0.4 \mathrm{eV}$ [Fig. 7(c)], the positive (negative) bias shifts the peak of $T^{\mathrm{R}}(E)$ to higher (lower) energy, while it suppresses (enhances) the negative dip of $T_{\perp}^{\mathrm{L}}(E)$, giving rise to $d T_{\perp} / d V>0$ in the low-bias regime.

Finally, in Fig. 9 we display $\ln T_{\|}$and $\ln T_{\perp}$, normalized

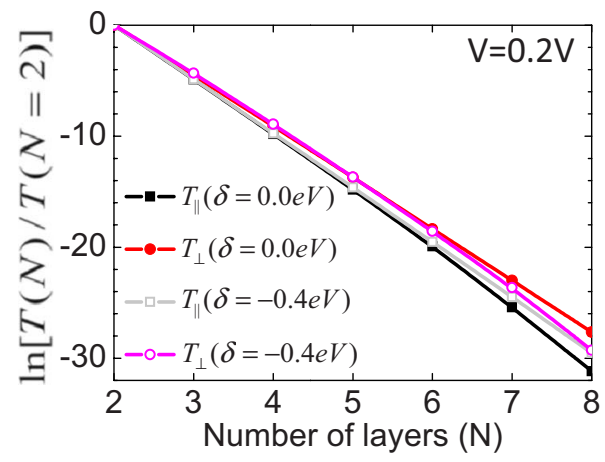

FIG. 9. (Color online) $\ln T_{\|}$and $\ln T_{\perp}$, normalized to their values for the two-layer barrier thickness, versus the number of barrier layers, $N$, for $V=0.2 \mathrm{~V}$ and various values of $\delta$. 
to their values for the two-layer barrier thickness as a function of the number of barrier layers, $N$, for $0.2 \mathrm{~V}$ bias and for $\delta=0.0$ and $-0.4 \mathrm{eV}$. Slonczewski ${ }^{6}$ has shown that at zero bias and for a free-electron system where the tunneling is dominated around the $\Gamma$ point, both spin torque components have the same exponential decay with the barrier thickness, $d=N a$, of the form, $T_{\|}=A e^{-2 \kappa_{\|} d}$ and $T_{\perp}=B e^{-2 \kappa_{\perp} d}$. Here $\kappa_{\|}$ $=\kappa_{\perp}$, and $A$ and $B$ are complicated functions of the energy parameters. Since in the simple-cubic TB model employed here the transport is determined from the entire twodimensional Brillouin zone, the decay constants are $\kappa_{\|} a$ $=2.58$ and $\kappa_{\perp} a=2.30$ for $\delta=0.0 \mathrm{eV}$ and $\kappa_{\|} a=2.45$ and $\kappa_{\perp} a=2.42$ for $\delta=-0.4 \mathrm{eV}$.Unlike spin valves, where $T_{\perp}$ $\ll T_{\|}, T_{\perp} \approx T_{\|}$in MTJ, thus playing also an important role in the CIMR. ${ }^{3-5}$

\section{CONCLUSION}

In conclusion, we have employed tight-binding calculations and the nonequilibrium Keldysh formalism to study the effect of asymmetry in BF between the left and right FM leads on the bias behavior of TMR, $T_{\|}$, and $T_{\perp}$. We have shown that the universal expression (18) relating the spintransfer torque, $T_{\|}$, with the spin currents of the FM and AF configurations is valid also for asymmetric MTJ. The decrease (increase) in the BF of the right FM lead compared to that of the left lead shifts the maximum of the TMR to negative (positive) bias. The asymmetry-induced change in the bias dependence of TMR and $T_{\|}$can be understood on the basis of the Brinkman tunnel model. The exponential decrease in $T_{\|}$with barrier width is more pronounced than that of $T_{\perp}$.

The asymmetry in BF has a dramatic effect on the bias behavior of $T_{\perp}$, which can vary from linear to quadratic bias dependence, with positive or negative curvature at low bias. We have derived a general expression [Eq. (19)] relating $T_{\perp}$ with four independent NEIECs, $J_{\mathrm{FM}}^{\sigma \sigma^{\prime}}$, associated with the majority- and minority-spin channels, $\sigma, \sigma^{\prime}=\uparrow, \downarrow$, of the two leads solely in the FM configuration. The underlying mechanism is the bias-induced change in the energy dependence of the left and right reservoir contributions, $T_{\perp}^{\mathrm{L}}(E)$ and $T_{\perp}^{\mathrm{R}}(E)$, to the net $T_{\perp}$. The bias behavior of the NEIEC components can be selectively tuned with the BF of the free and pinned FM layers, thus opening a new avenue for controlling experimentally $T_{\perp}$. These results may serve as simple guiding rules for future experimental studies of the NEIEC in MTJ via controlled fabrication of the FM leads.

\section{ACKNOWLEDGMENTS}

We thank E. Y. Tsymbal, M. Chshiev, D. C. Ralph, H. Kubota, M. Stiles, and P. Haney for useful conversations. The work at California State University, Northridge was supported by NSF-PREM under Grant No. DMR-00116566. The work at Princeton University was supported by DOE under Grant No. DE-FG02-05ER46201.
${ }^{1}$ A. A. Tulapurkar, Y. Suzuki, A. Fukushima, H. Kubota, H. Maehara, K. Tsunekawa, D. D. Djayaprawira, N. Watanabe, and S. Yuasa, Nature (London) 438, 339 (2005).

${ }^{2}$ G. D. Fuchs, J. A. Katine, S. I. Kiselev, D. Mauri, K. S. Wooley, D. C. Ralph, and R. A. Buhrman, Phys. Rev. Lett. 96, 186603 (2006).

${ }^{3}$ J. C. Sankey, Y.-T. Cui, J. Z. Sun, J. C. Slonczewski, R. A. Buhrman, and D. C. Ralph, Nat. Phys. 4, 67 (2008).

${ }^{4}$ H. Kubota, A. Fukushima, K. Yakushiji, T. Nagahama, S. Yuasa, K. Ando, H. Maehara, Y. Nagamine, K. Tsunekawa, D. D. Djayaprawira, N. Watanabe, and Y. Suzuki, Nat. Phys. 4, 37 (2008).

${ }^{5}$ S. Petit, C. Baraduc, C. Thirion, U. Ebels, Y. Liu, M. Li, P. Wang, and B. Dieny, Phys. Rev. Lett. 98, 077203 (2007).

${ }^{6}$ J. C. Slonczewski, Phys. Rev. B 39, 6995 (1989).

${ }^{7}$ J. C. Slonczewski, Phys. Rev. B 71, 024411 (2005).

${ }^{8}$ I. Theodonis, N. Kioussis, A. Kalitsov, M. Chshiev, and W. H. Butler, Phys. Rev. Lett. 97, 237205 (2006).

${ }^{9}$ C. Heiliger and M. D. Stiles, Phys. Rev. Lett. 100, 186805 (2008).

${ }^{10}$ J. Xiao, G. E. W. Bauer, and A. Brataas, Phys. Rev. B 77, 224419 (2008).

${ }^{11}$ S. S. P. Parkin, C. Kaiser, A. Panchula, P. M. Rice, B. Hughes, M. Samant, and S.-H. Yang, Nature Mater. 3, 862 (2004).

${ }^{12}$ S. Yuasa, T. Nagahama, A. Fukushima, Y. Suzuki, and K. Ando, Nature Mater. 3, 868 (2004).

${ }^{13}$ A. Kalitsov, M. Chshiev, I. Theodonis, N. Kioussis, and W. H.
Butler, Phys. Rev. B 79, 174416 (2009).

${ }^{14}$ C. W. Miller, I. K. Schuller, R. W. Dave, J. M. Slaughter, Y. Zhou, and J. Åkerman, J. Appl. Phys. 103, 07 A904 (2008).

${ }^{15}$ Y.-Z. Liu, W. G. Wang, T. Moriyama, J. Q. Xiao, and Z. Zhang, Phys. Rev. B 75, 134420 (2007).

${ }^{16}$ P. H. P. Koller, H. J. M. Swagten, W. J. M. de Jonge, H. Boeve, and R. Coehoorn, Appl. Phys. Lett. 84, 4929 (2004).

${ }^{17}$ H. Brückl, J. Schmalhorst, G. Reiss, G. Gieres, and J. Wecker, Appl. Phys. Lett. 78, 1113 (2001).

${ }^{18}$ P. LeClair, J. T. Kohlhepp, C. H. van de Vin, H. Wieldraaijer, H. J. M. Swagten, W. J. M. de Jonge, A. H. Davis, J. M. MacLaren, J. S. Moodera, and R. Jansen, Phys. Rev. Lett. 88, 107201 (2002).

${ }^{19}$ F.-f. Li, Z.-z. Li, M.-w. Xiao, J. Du, W. Xu, and A. Hu, Phys. Rev. B 69, 054410 (2004).

${ }^{20}$ E. Y. Tsymbal, A. Sokolov, I. F. Sabirianov, and B. Doudin, Phys. Rev. Lett. 90, 186602 (2003).

${ }^{21}$ M. Sharma, S. X. Wang, and J. H. Nickel, Phys. Rev. Lett. 82, 616 (1999).

${ }^{22}$ M. Wilczyński, J. Barnaś, and R. Świrkowicz, Phys. Rev. B 77, 054434 (2008).

${ }^{23}$ Y.-H. Tang, N. Kioussis, A. Kalitsov, W. H. Butler, and R. Car, Phys. Rev. Lett. 103, 057206 (2009).

${ }^{24}$ W. F. Brinkman, R. C. Dynes, and J. M. Rowell, J. Appl. Phys. 41, 1915 (1970).

${ }^{25}$ Z. Li, S. Zhang, Z. Diao, Y. Ding, X. Tang, D. M. Apalkov, Z. 
Yang, K. Kawabata, and Y. Huai, Phys. Rev. Lett. 100, 246602 (2008)

${ }^{26}$ C. Caroli, R. Combescot, P. Nozieres, and D. Saint-James, J. Phys. C 4, 916 (1971).
${ }^{27}$ I. Theodonis, A. Kalitsov, and N. Kioussis, Phys. Rev. B 76, 224406 (2007).

${ }^{28}$ Y.-H. Tang, N. Kioussis, A. Kalitsov, W. H. Butler, and R. Car, J. Phys.: Conf. Ser. (to be published). 